\title{
Socioeconomic status and use of analgesic drugs before and after primary hip arthroplasty: a population-based cohort study of 103,209 patients during 1996-2018
}

\author{
André N KLENØ ${ }^{1}$, Martin B STISEN ${ }^{1}$, Nina M EDWARDS ${ }^{1}$, Inger MECHLENBURG ${ }^{2-4}$, and Alma B PEDERSEN ${ }^{1,3}$
}

\author{
${ }^{1}$ Department of Clinical Epidemiology, Aarhus University Hospital, Aarhus N; ${ }^{2}$ Department of Or-thopaedic Surgery, Aarhus University Hospital, Aarhus N; \\ ${ }^{3}$ Department of Clinical Medicine, Aar-hus University, Aarhus N; 4 Department of Public Health, Aarhus University Aarhus, Denmark \\ Correspondence: ank@clin.au.dk \\ Submitted 2021-07-05. Accepted 2021-11-18.
}

Background and purpose - Total hip arthroplasty (THA) is an effective and common procedure. However, persistent pain and analgesic requirement up to 2 years after THA surgery are common. We examined the trends in the utilization of analgesics before and after THA, overall, and in relation to socioeconomic status (SES) in a populationbased cohort.

Patients and methods - We used the Danish Hip Arthroplasty Register to identify 103,209 patients who underwent THA between 1996 and 2018. Data on prescriptions and SES markers was obtained from Danish medical databases. Prevalence rates of redeemed prescriptions for analgesics with $95 \%$ confidence intervals were calculated for 4 quarters before and 4 quarters after THA for the entire THA population, and by 3 SES markers (education, cohabiting status, and wealth).

Results - Overall, the prevalence of analgesic use prior to surgery was $42 \%$ at $9-12$ months and $59 \%$ at $0-3$ months before the THA. The prevalence of analgesics reached its highest at 64\% 0-3 months after THA but declined to $27 \%$ at 9-12 months after THA. Low education, living alone, and having low wealth (low SES) were associated with higher prevalence of analgesics use both before and after THA.

Interpretation - 59\% of patients used analgesics 0-3 months before surgery, which could indicate that THA might not be considered the last option for treatment and that surgery criteria might depend more on factors such as patient preferences or hip function. Moreover, health professionals should prioritize the use of a detailed plan when phasing out analgesics after THA to counteract unnecessary use, especially when treating patients with low SES.
Treatment of hip osteoarthritis (OA) includes several modalities: non-pharmacological involving weight loss and patient education (1); pharmacological involving non-opioid or opioid analgesics; and surgery, when other treatment modalities have failed to provide adequate pain relief (2). Nonetheless, a Finnish study found that only $48 \%$ of patients undergoing total hip replacement (THA) consumed analgesics $0-3$ months prior to surgery (3). In addition, a systematic review by Beswick et al. showed that $7-23 \%$ of patients undergoing THA experienced long-term postoperative joint pain, along with other studies suggesting high consumption of analgesics 1-2 years postTHA (4,5).

In Denmark, the number of THAs almost doubled from 2000 to 2018, and an increasing trend is seen in other western countries as well $(2,6)$. Thus, there is a discrepancy between the guidelines for treatment of hip OA, threshold for when THA is indicative, and success of THA surgery measured by pain reduction, and analgesics use. It is unclear which factors contribute to this discrepancy.

An American study found patients with low education experienced more severe hip OA, and a Danish study found that residents with low education and income were more likely to undergo THA $(\mathbf{7 , 8})$. Furthermore, a Norwegian study found that divorce, low income, and low educational level were associated with persistent opioid use (9). However, it is generally unclear whether socioeconomic status (SES) is associated with pain measured by use of analgesics before and after THA.

Therefore, we conducted a population-based cohort study to examine the trends in utilization of analgesics before and after THA, overall, and in relation to markers of SES. 


\section{Patients and methods}

\section{Study design and population}

We conducted this population-based descriptive cohort study in Denmark, a country of 5.7 million residents, with universal access to healthcare, including partial reimbursement of prescription drug cost. Patients undergoing THA due to OA from 1996 to 2018 were identified in the Danish Hip Arthroplasty Register (DHR) ( $\mathrm{n}=103,209$ patients). All public orthopedic departments and private hospitals in Denmark performing THA report to the register, and the registration completeness for primary THA is $95 \%(\mathbf{1 0 , 1 1 )}$. A unique civil registration (CPR) number, recorded in the Civil Registration System (CRS), has been assigned to every Danish citizen and resident since 1968. The CPR encodes age and sex, which enables us to link individual-level data between multiple databases.

\section{Socioeconomic status markers}

Data on markers of SES including education level and wealth were obtained from Statistics Denmark, which is a nationwide database updated yearly and containing detailed individuallevel SES information on all Danish residents (12). Data on cohabiting status was obtained from the CRS.

Information on SES markers was obtained for each patient undergoing THA at the time of operation.

Educational level was assessed as highest education obtained and divided into 3 categories: low, defined as high school as highest completed education, medium, defined as vocational education or higher general and preparatory examination programs, and high, defined as bachelor's degree or higher.

Cohabiting status was categorized as living alone or cohabiting (married or living with a partner). Patients with other cohabiting status were excluded from the analysis.

Wealth was generated using family income or family liquid assets. Under the assumption that income would not be an accurate measurement for patients who were retired, family liquid assets was used for patients with aged $\geq 65$ years (age of retirement in Denmark) and family income was used for patients with aged $<65$ years $(7)$. To account for yearly variation a 5 -year time span was applied for both family income and family liquid assets, and the estimate used in this study was based on the average of the 5 most recent years of data before THA. Both family income and family liquid assets were categorized into tertiles low, medium, and high, and combined into the wealth variable.

\section{Analgesics utilization}

Prescriptions for analgesics use were identified in the Danish National Prescription Registry, which was established in 1994, and contains detailed individual-level information on all prescriptions redeemed by Danish residents in the community pharmacies, excluding hospital dispensaries since 1995 (13). The registry contains the Anatomical Therapeutic Classification System (ATC) codes and the dispensation date.
We defined any analgesics drugs, as well as NSAIDs and opioids, separately. Data from 4 quarters before THA and 4 quarters after THA was obtained, and referred to as quarter -4 (9-12 months prior), quarter -3 (6-9 months prior), quarter -2 (3-6 months prior, quarter -1 ( $0-3$ months prior) etc. (3). In each quarter, patients were defined as a user of any analgesic drugs if they redeemed 1 or more prescriptions for either NSAID or opioids, and non-users if they did not. Likewise, we defined NSAIDs and opioids.

To define use of NSAID, we used ATC code M01A. To define use of opioids, we used the following ATC codes: N02AA01, N02AB03, N02AA03, N02AG02, N07BC02, N02AA04, N02AA05, N02AB02, N02AA55, N02AX02, N02AX06, N02A301, R05DA04, N02AJ06 (Table 1, see Supplementary data).

\section{Patient characteristics}

Information on age and sex at the time of THA was collected from the CRS (14).

Comorbidities from 10 years prior to THA for all patients were collected from the Danish National Patient Registry (DNPR) (15). This contains data on all somatic inpatient contacts since 1977 and on all outpatient and emergency room contacts since 1995 .

Comorbidities at the time of THA were measured using the Charlson Comorbidity Index (CCI), based on ICD-10 codes adapted for administrative purposes (16). The CCI was divided into 3 categories: low, defined as no comorbidities, medium, defined as 1 or 2 comorbidities, and high, defined as 3 or more comorbidities.

\section{Statistics}

We calculated prevalence rates with $95 \%$ confidence intervals (CI) of any analgesic drug users within each of the 4 quarters before and after THA for the entire study population. For each quarter, the prevalence was calculated as the number of patients who had redeemed at least 1 prescription for any analgesics divided by the total number of patients still at risk. Differences in prevalence rates are presented in percentage points (pp). Prevalence rates were calculated in the same way separately for NSAIDs and opioids, and stratified by SES marker, education, cohabiting status, and wealth. The results are presented in table form and illustrated graphically.

In addition, we conducted a sensitivity analysis by calculating the prevalence of opioid use after surgery among patients who had not redeemed any prescriptions for opioids in the year prior to surgery (opioid-naive patients).

The analyses were performed using Stata 16.0 (StataCorp, College Station, TX, USA) (17).

\section{Missing data}

Information on age, sex, and comorbidities for patients with missing data on education and patients with cohabiting status category other are presented in Table 2. Patient characteris- 
tics for patients with missing data on wealth were left out due to low numbers.

\section{Ethics, funding, and potential conflicts of interest}

According to Danish law, ethics committee approval is not required for registry-based studies. The study was approved by the Danish Data Protection Agency's journal number 2015-57-0002 and Aarhus University record number 2016-051-000001, id.nr. 880. This research was partly funded by the Orthopedic-Surgical Research Foundation, Aarhus The authors report no conflict of interest.

\section{Results}

From 1996 to 2018, we identified 105,518 patients with hip OA undergoing primary THA. 2,309 patients were excluded for undergoing revision THA within 1 year after their primary THA, and the final study population included 103,209 patients (Figure 1). Patients were predominantly female (56\%), mean age was 69 years (15-99), and the majority had no comorbidities (79\%). Patient characteristics by SES markers are presented in Table 2.

\section{Overall use of analgesic drugs}

The prevalence of patients using any analgesics was high before THA, being $42 \%$ in quarter -4 and $59 \%$ in quarter -1 .
The prevalence was highest at $64 \%$ in quarter 1 but decreased to $27 \%$ in quarter 4 . Prevalence rates of opioid users was $15 \%$ in quarter $-4,31 \%$ in quarter -1 , and decreased to $14 \%$ in quarter 4. Prevalence of opioid use peaked in quarter 1 after surgery by $50 \%$ and, overall, the prevalence of opioid users in quarter $4(14 \%)$ was similar to the prevalence of opioid users in quarter -4 (15\%) (Table 3). The overall most prescribed opioid was tramadol, which accounted for 52\% (quarter -4 ), $37 \%$ (quarter 1), and $43 \%$ (quarter 4). During quarter 1 the prevalence of patients using oxycodone increased and accounted for $34 \%$.

Table 2. Patient characteristics

\begin{tabular}{|c|c|c|c|c|c|c|c|c|c|c|c|}
\hline \multirow[b]{2}{*}{ Factor } & \multicolumn{5}{|c|}{ Education } & \multicolumn{3}{|c|}{ Cohabiting status } & \multicolumn{3}{|c|}{ Wealth } \\
\hline & $\begin{array}{c}\text { Total } \\
\mathrm{n}\end{array}$ & $\begin{array}{c}\text { Low } \\
n=42,394\end{array}$ & $\begin{array}{c}\text { Medium } \\
\mathrm{n}=37,532\end{array}$ & $\begin{array}{c}\text { High } \\
\mathrm{n}=16,128\end{array}$ & $\begin{array}{c}\text { Missing } \\
\mathrm{n}=7,155\end{array}$ & $\begin{array}{c}\text { Alone } \\
\mathrm{n}=34,084\end{array}$ & $\begin{array}{l}\text { Cohabiting } \\
\mathrm{n}=62,316\end{array}$ & $\begin{array}{c}\text { Other } \\
n=6,809\end{array}$ & $\begin{array}{c}\text { Low } \\
\mathrm{n}=34,393\end{array}$ & $\begin{array}{c}\text { Medium } \\
\mathrm{n}=34,391\end{array}$ & $\begin{array}{c}\text { High } \\
\mathrm{n}=34,391\end{array}$ \\
\hline \multicolumn{12}{|l|}{ Sex } \\
\hline Male & 45,545 & $16,899(40)$ & $19,388(52)$ & 6,581 (41) & 2,677 (37) & 8,905 (26) & 33,206 (53) & $3,434(50)$ & 12,049 (35) & $15,930(46)$ & $17,551(51)$ \\
\hline Female & 57,664 & 25,495 (60) & 18,144 (48) & 9,547 (59) & 4,478 (63) & 25,179 (74) & $29,110(47)$ & $3,375(50)$ & $22,344(65)$ & $18,416(54)$ & $16,840(49)$ \\
\hline \multicolumn{12}{|l|}{ Age } \\
\hline \multicolumn{2}{|c|}{ Mean (SD) } & $71(8.9)$ & $67(9.8)$ & $67(9.5)$ & $81(8.5)$ & $73(9.9)$ & $68(10)$ & $66(10)$ & $70(9.9)$ & $69(9.8)$ & $68(10)$ \\
\hline$<45$ & 1,773 & 399 (1) & 981 (3) & 353 (2) & 40 (1) & 416 (1) & 1,195 (2) & $162(2)$ & $471(1)$ & $572(2)$ & 727 (2) \\
\hline $45-55$ & 8,509 & $2,357(6)$ & 4,362 (12) & 1,658 (10) & $132(2)$ & 1,749 (5) & $5,839(9)$ & 921 (14) & $2,118(6)$ & 2,675 (8) & 3,712 (11) \\
\hline $56-65$ & 25,512 & $8,964(21)$ & $11,358(30)$ & 4,853 (30) & 337 (5) & $5,476(16)$ & $17,759(29)$ & 2,277 (33) & $9,065(27)$ & $8,778(25)$ & $7,653(22)$ \\
\hline $66-75$ & 40,171 & $18,700(44)$ & 14,455 (38) & $6,405(40)$ & $611(8)$ & $12,176(36)$ & $25,756(41)$ & 2,239 (33) & 12,017 (35) & 13,437 (39) & 14,714 (43) \\
\hline $76-85$ & 23,975 & $11,014(26)$ & 5,930 (16) & 2,639 (17) & $4,392(61)$ & 11,930 (35) & $10,978(18)$ & $1,067(16)$ & $9,232(27)$ & $7,920(23)$ & $6,815(20)$ \\
\hline$>85$ & 3,269 & 960 (2) & 446 (1) & $220(1)$ & $1,643(23)$ & $2,337(7)$ & 789 (1) & $143(2)$ & $1,490(4)$ & 1,009 (3) & 770 (2) \\
\hline \multicolumn{12}{|l|}{$\mathrm{CCl}$} \\
\hline Low & 87,046 & 32,94 & $30,109(80)$ & 13,3 & 77) & 25,9 & 50 & 5 , & 26,3 & $4(79)$ & $7(82)$ \\
\hline Medium & 18,920 & $7,963(19)$ & 6,275 (17) & 2,437 (15) & $1,430(20)$ & $6,857(20)$ & $10,174(16)$ & $1,074(16)$ & 6,715 (19) & 6,035 (18) & 5,347 (15) \\
\hline High & 3,370 & $1,486(3)$ & $1,148(3)$ & $390(2)$ & 201 (3) & 1,287 (4) & $1,771(3)$ & $167(2)$ & $1,306(4)$ & 1,012 (3) & 907 (3) \\
\hline
\end{tabular}

Characteristics on missing data concerning wealth is left out due to a low number of patients $(n=34)$.

$\mathrm{CCl}=$ Charlson Comorbidity Index. 
Table 3. Prevalence (\%) of analgesic drugs users with $(95 \% \mathrm{Cl})$

\begin{tabular}{|c|c|c|c|c|c|c|c|c|}
\hline \multirow[b]{2}{*}{ Factor } & \multicolumn{4}{|c|}{ Prior to surgery } & \multicolumn{4}{|c|}{ Post-surgery } \\
\hline & $-\mathrm{Q} 4$ & $-\mathrm{Q3}$ & $-\mathrm{Q} 2$ & $-\mathrm{Q} 1$ & Q1 & Q2 & Q3 & Q4 \\
\hline \multicolumn{9}{|c|}{ Analgesic drug use } \\
\hline All & $41.8(40.5-42.1)$ & $46.8(46.5-47.1)$ & $54.7(54.4-55.0)$ & $58.8(58.5-59.1)$ & $64.0(63.7-64.3)$ & $29.7(29.4-30.0)$ & $28.0(27.7-28.2)$ & $27.0(26.8-27.3)$ \\
\hline NSAID & $33.8(33.5-34.1)$ & $37.8(37.5-38.1)$ & $43.1(42.8-43.4)$ & $43.2(42.9-43.5)$ & $31.5(31.2-31.7)$ & $19.0(18.8-19.2)$ & $18.1(17.8-18.3)$ & $17.4(17.2-17.7)$ \\
\hline Opioids & $14.6(14.3-14.8)$ & $17.3(17.1-17.6)$ & $23.2(22.9-23.5)$ & $31.0(30.7-31.3)$ & $49.8(49.5-50.1)$ & $15.7(15.5-16.0)$ & $14.4(14.2-14.6)$ & $13.9(13.7-14.1)$ \\
\hline \multicolumn{9}{|l|}{ Education level } \\
\hline Low & $44.9(44.4-45.4)$ & $49.7(49.3-50.2)$ & $57.8(57.3-58.3)$ & $61.0(60.6-61.5)$ & $63.4(62.9-63.9)$ & $32.3(31.8-32.7)$ & $30.8(30.4-31.2)$ & $29.6(29.2-30.1)$ \\
\hline Medium & $39.4(39.0-39.9)$ & $44.6(44.1-45.1)$ & $52.5(52.0-53.0)$ & $57.6(57.1-58.1)$ & $67.2(66.7-67.7)$ & $28.5(28.1-29.0)$ & $26.6(26.2-27.1)$ & $25.7(25.3-26.2)$ \\
\hline High & $36.1(35.3-36.8)$ & $40.6(39.9-41.4)$ & $48.5(47.7-49.3)$ & $53.8(53.0-54.6)$ & $64.7(64.0-65.5)$ & $25.0(24.4-25.7)$ & $22.4(21.8-23.1)$ & $21.8(21.2-22.5)$ \\
\hline \multicolumn{9}{|c|}{ Cohabiting status } \\
\hline Alone & $45.5(45.0-46.0)$ & $50.8(50.3-51.4)$ & $58.1(57.6-58.6)$ & $61.6(61.1-62.2)$ & $63.6(63.1-64.1)$ & $32.9(32.4-33.4)$ & $31.3(30.8-31.8)$ & $30.3(29.8-30.8)$ \\
\hline Cohabiting & $39.7(39.3-40.1)$ & $44.6(44.2-44.9)$ & $52.9(52.5-53.2)$ & $57.2(56.8-57.6)$ & $64.2(63.8-64.6)$ & $28.1(27.7-28.4)$ & $26.1(25.8-26.5)$ & $25.1(24.8-25.5)$ \\
\hline \multicolumn{9}{|l|}{ Wealth } \\
\hline Low & $49.3(4$ & $54.5(54$. & ) & 63.9( & $63.0(62$. & $36.0(35.5$ & 34.3( & 33.3 \\
\hline Medium & $41.8(41.2-42.3)$ & $46.8(46.3-47.3)$ & $55.2(54.7-55.7)$ & $59.0(58.5-59.6)$ & $62.4(61.9-62.9)$ & $29.1(28.6-29.6)$ & $27.4(26.9-27.9)$ & $26.5(26.0-27.0)$ \\
\hline High & $34.3(33.8-34.8)$ & $39.0(38.5-39.5)$ & $46.8(46.3-47.3)$ & $53.3(52.8-53.9)$ & $66.6(66.1-67.1)$ & $24.1(23.7-24.6)$ & $21.9(22.0-22.8)$ & $21.4(21.0-21.9)$ \\
\hline
\end{tabular}

Each quarter $(Q)$ is equivalent to a period of 3 months and the total timespan is 2 years, ranging from 1 year prior to surgery to 1 year post-surgery. Prevalence of analgesic drug use among SES markers is based on patients redeeming any analgesics.
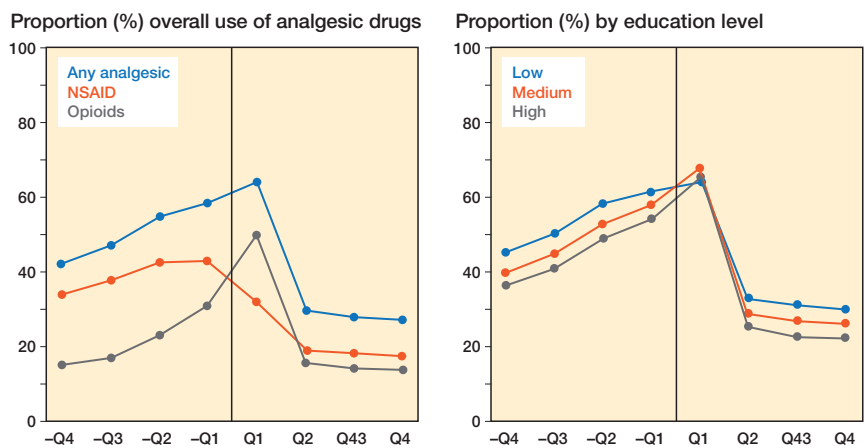

Proportion (\%) by cohabiting status
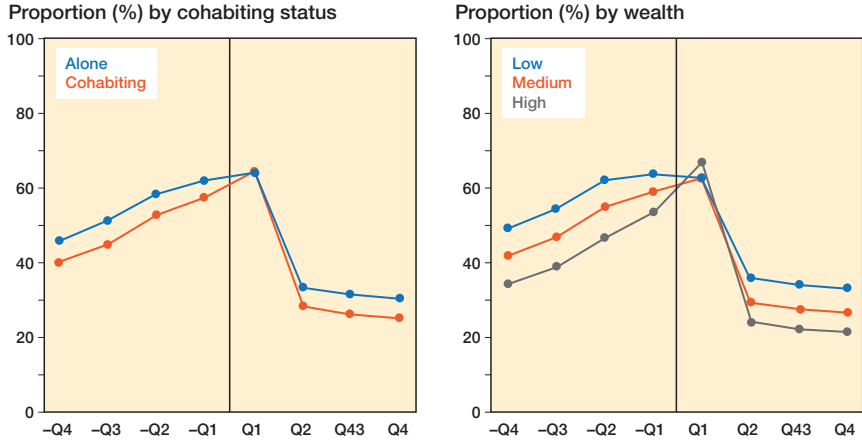

Figure 2. Prevalence of analgesic drugs users one year before and one year after surgery overall and by SES markers.

Among opioid-naive patients, the prevalence of opioid users was $41 \%$ in quarter 1 . In quarter 2 it decreased drastically, and finished at $5 \%$ in quarter 4.

Prevalence rates of NSAID user were $34 \%$ in quarter -4 and highest at $43 \%$ in quarter -1 , as opposed to opioid use, which peaked in quarter 1 . In quarter 1 the prevalence of NSAID use decreased to $32 \%$ and continued to decrease to quarter 4 where the prevalence was $17 \%$ (Figure 2 and Table 3 ). The most prescribed NSAID overall was ibuprofen, which accounted for $37 \%$ (quarter -4 ), $54 \%$ (quarter 1 ), and $41 \%$ (quarter 4). Diclofenac accounted for $18 \%$ (quarter -4 ), $10 \%$ (quarter 1), and 13\% (quarter 4).

\section{Educational level}

Data from 96,054 patients was assessable on education level. Among patients with medium education $50 \%$ were female, and for patients with low or high education $60 \%$ were female. Patients with low education were older and there was a slightly higher prevalence of patients with comorbidities compared with patients with high education (Table 2).

The prevalence rates of analgesics users were higher among low education than high education throughout all quarters before THA and quarters 2, 3, and 4 after THA. Observed between-group differences were $9 \mathrm{pp}$ in quarter -4 and $8 \mathrm{pp}$ in quarter 4 (Figure 2 and Table 3).

However, all educational groups experienced an approximately 15 pp decrease in use of analgesics over the total time span of the study and slightly more than $30 \mathrm{pp}$ from the quarter -1 to the quarter 4 .

\section{Cohabiting status}

Data on cohabiting status was analyzed from 96,400 patients. Among patients who were cohabiting, sex distribution was approximately $50 \%$ for both men and woman, while $74 \%$ of patients living alone were female. Patients living alone were more prevalent in age groups $>75$ years and had slightly more comorbidities compared with patients who were cohabiting. Patients categorized as other were similar overall to patients categorized as cohabiting.

Prevalence rates of analgesics users were higher throughout all quarters before THA and quarters 2, 3, and 4 after THA among patients living alone than for patients who were cohabiting. Between group differences were $6 \mathrm{pp}$ in quarter -4 and 
5 pp in quarter 4 (Figure 2 and Table 3). Overall, a decrease in use of analgesics was observed from quarter -4 to quarter 4 regardless of cohabiting status.

\section{Wealth}

Data from 103,175 patients was assessable on wealth. Among patients with high wealth, sex distribution was approximately $50 \%$ for both male and female. $54 \%$ of patients were female in the medium-wealth group and $65 \%$ of patients were female in the low-wealth group. Patients with low wealth were marginally older and had slightly more comorbidities than patients with high wealth.

Prevalence rates of analgesics users were higher among patients with low wealth than patients with high wealth throughout all quarters before THA and quarters 2, 3, and 4 after THA. Between-group differences were $15.0 \mathrm{pp}$ in quarter -4 and 12 pp in quarter 4 (Figure 2 and Table 3 ).

\section{Discussion}

Use of analgesics increased in the year prior to THA and was highest at 64\% 0-3 months after. Prevalence rates decreased after surgery, but approximately one-fourth kept using analgesics. The low SES stratifications were associated with higher prevalence of analgesics users before and after THA compared with high SES. Wealth was the most influential factor in the association between analgesic drug use and SES. 1 year post-THA, the prevalence was lower than 1 year before THA across all SES groups.

\section{Comparison with other studies}

To our knowledge, no other studies have investigated analgesic drug use in patients undergoing THA while focusing on the relation to SES.

\section{Overall use of analgesic drugs}

In line with our estimates for the overall use of analgesics, a study by Blågestad et al. found similar results in patients undergoing THA in Norway (5). They found the prevalence of analgesics use to be $49 \%$ in quarter -4 , which was higher than $42 \%$ in our study. The $59 \%$ in quarter -1 was almost identical to what we found, and the $33 \%$ in quarter 4 was higher than the $27 \%$ in our study. Furthermore, a Finnish study by Rajamäki et al. showed overall use of analgesics increasing from approximately $40 \% 1$ year prior to surgery, decreasing to a prevalence of around $25 \% 1$ year post-surgery (3).

We found that $5 \%$ of opioid-naive patients were using opioids 1 year after THA. Conversely, an American study suggests that this is applicable to $10 \%$ of patients (18). To minimize false classifications of opioid users, patients were excluded for undergoing revision in our study and for undergoing any subsequent operation in the study by Cook et al. However, it is not certain whether patients were taking opi- oids in relation to other conditions such as cancer, or if THA resulted in subsequent long-term use. In general, the study by Cook et al. supports our findings despite a higher percentage of opioid users among opioid-naive patients. The differences are possibly a result of developing guidelines for opioid use, where, due to intensive national and international focus on the problem, Denmark might be a bit further on in the process of implementing these than the USA (19). 5\% might be seen as a fairly low estimate, but clinically highly relevant due to the side effects long-term opioid use may have on quality of life and pain.

\section{SES markers}

A systematic review by Hernández et al. from 2015 investigated pre-surgical predictors for post-surgical pain after THA or total knee arthroplasty (20). The majority of patients were $>60$ years and had OA, making the study population comparable to our study. Reviewing 37 studies, they found a strong association between low SES and post-surgical pain.

Svendsen et al. investigated characteristics in short- and long-term opioid use in Norway in a study population of 229,174 patients (9). They reported that having low education, being divorced, or having low income was associated with long-term opioid use. Although the included patients were slightly younger than in our study and other patient groups than those with OA were included, the results of the comprehensive study support our overall findings.

A study by Grol-Prokopczyk investigated demographic characteristics for persistent opioid use among elderly American residents (21). They found that low wealth was a strong consistent predictor of opioid use, which is in line with our results. However, in contrast to our results, Grol-Prokopczyk did not find a considerable difference in opioid use among different education levels. Although SES is a commonly used term, underlying reasonings for the associations should be drawn regarding the specific categories. The associations between SES categories and use of analgesics are based on different behavioral patterns, from which we can draw different assumptions as to why they emerge. The fact that more patients with low education are likely to use analgesics might be associated with a lower threshold for seeking medical attention (22). Moreover, patients living alone could be experiencing a feeling of loneliness and thus be more exposed to having an overconsumption of analgesics (23).

Overall, the results from our study generally align with existing evidence and furthermore contribute with new knowledge on an as yet uninvestigated matter.

\section{Strengths and limitations}

This nationwide cohort study contributes with a large sample size of 103,209 patients with data from 24 years of registry data. With the DHR having a high completeness of THA registrations we were able to include complete follow-up on all THAs, along with information on death for valid calculations 
on prevalence rates of analgesics users. Furthermore, Statistics Denmark contains detailed individual-level information on SES, which overall enables us to contribute with a highly accurate description of the Danish population.

A potential limitation to this study is lack of information on the indication for the prescription of analgesics; thus, patients may receive analgesic drugs for health-related problems other than hip OA. However, comorbidities were almost evenly distributed among SES categories and the majority of patients had no comorbidities at the time of THA, suggesting that patients generally should not receive a substantial amount of analgesics for purposes other than treating OA during a short period prior to surgery and post-surgery. In addition, a Danish study suggests that $50 \%$ of patients receive more doses of opioids than needed, which might result in them not using all their dispensed analgesics (24). It is not possible to ensure $100 \%$ correct classification of drug exposure when using large databases, but this method is considered superior to, e.g., selfreported data (25).

Furthermore, it is not certain that low SES is causing more patients to use analgesics. The observed analgesic patterns can potentially be explained by lifestyle-related factors such as obesity and BMI. It has been shown that obese patients especially are exposed to pain due to increased load on their joints, which might be part of the reason why patients are using analgesics in OA treatment (26). Data on patients' BMI was not available in this study but would be an interesting addition to patient characteristics because it may contribute to patients remaining as analgesics users after THA. Likewise, other unavailable measurements like patient-reported outcomes or radiographically assessed hip OA would add additional complexity to the analysis, as other researchers found that more severe degrees of pre-operative radiologically assessed OA were associated with higher postoperative pain reduction (27) and that lower SES was associated with more severe OA (8).

\section{Conclusion}

In conclusion, this study contributes with a comprehensive cohort of 103,209 patients undergoing THA. Use of analgesics in THA patients decreased 9-12 months after surgery, but approximately one-fourth continued to use analgesics. Markers of low SES were associated with higher use of analgesics both before and after THA, and lower reduction in use of analgesics after THA compared with markers of high SES.

The increasing trend in utilization of analgesic drugs in the year prior to surgery aligns with the clinical aspect of optimizing medication before surgery, and the decrease in redeemed prescriptions confirms a reduced demand for analgesics after THA. With a prevalence of $59 \%$ of patients using analgesics 0-3 months before surgery, this could indicate that there is a basis for optimizing medical treatment. However, it can also be an indication that surgical criteria have developed over time, with the general perception that a patient's functional level before THA is a determining factor in the outcome of their rehabilitation. In addition, health professionals should prioritize the use of a detailed plan when phasing out analgesics after THA to counteract unnecessary use. This might be especially beneficial when treating patients with low SES due to several studies concluding that inequality in health is an ongoing problem.

\section{Supplementary data}

Table 1 is available as supplementary data.

ANK drafted the manuscript. MBS, NME, IM, and ABP conceived and designed the study, interpreted the results, and revised the manuscript.

Acta thanks Ken Iseri and Rob Nelissen for help with peer review of this study.

1. Mechlenburg I, Reimer L C U, Kjeldsen T, Frydendal T, Dalgas U. Exercise as medicine during the course of hip osteoarthritis. Exerc Sport Sci Rev 2021; 49: 77-87. doi: 101249/jes0000000000000249

2. Ferguson R J, Palmer A J, Taylor A, Porter M L, Malchau H, GlynJones S. Hip replacement. Lancet 2018; 392: 1662-71. doi: 101016/ s0140-6736(18)31777-x

3. Rajamaki T J Jr, Puolakka P A, Hietaharju A, Moilanen T, Jamsen E. Use of prescription analgesic drugs before and after hip or knee replacement in patients with osteoarthritis. BMC Musculoskelet Disord 2019; 20: 427. doi: 101186/s12891-019-2809-4

4. Beswick A D, Wylde V, Gooberman-Hill R, Blom A, Dieppe P. What proportion of patients report long-term pain after total hip or knee replacement for osteoarthritis? A systematic review of prospective studies in unselected patients. BMJ Open 2012; 2: e000435. doi: 101136/ bmjopen-2011-000435

5. Blågestad T, Nordhus I H, Grønli J, Engesæter L B, Ruths S, Ranhoff A H, et al. Prescription trajectories and effect of total hip arthroplasty on the use of analgesics, hypnotics, antidepressants, and anxiolytics: results from a population of total hip arthroplasty patients. Pain 2016; 157: 64351. doi: 101097/jpain0000000000000414

6. Danish Hip Arthroplasty Register Annual reports; 2021. Available from: http://danskhoftealloplastikregisterdk/en/publications/annualreports/

7. Edwards N M, Varnum C, Overgaard S, Pedersen A B. The impact of socioeconomic status on the utilization of total hip arthroplasty during 1995-2017: 104,055 THA cases and 520,275 population controls from national databases in Denmark. Acta Orthop 2020. Epub Oct 27. 101080/1745367420201840111: 1-5. doi: 101080/1745367420201840111

8. Cleveland R J, Schwartz T A, Prizer L P, Randolph R, Schoster B, Renner J B, et al. Associations of educational attainment, occupation, and community poverty with hip osteoarthritis. Arthritis Care Res (Hoboken) 2013; 65: 954-61. doi: 101002/acr21920

9. Svendsen K, Fredheim O M, Romundstad P, Borchgrevink P C, Skurtveit S. Persistent opioid use and socio-economic factors: a population-based study in Norway. Acta Anaesthesiol Scand 2014; 58: 437-45. doi: 101111/aas12281

10. Pedersen A, Johnsen S, Overgaard S, Søballe K, Sørensen H T, Lucht U. Registration in the Danish Hip Arthroplasty Registry: completeness of total hip arthroplasties and positive predictive value of registered diagnosis and postoperative complications. Acta Orthop Scand 2004; 75: 43441. doi: 101080/00016470410001213-1

11. Gundtoft P H, Varnum C, Pedersen A B, Overgaard S. The Danish Hip Arthroplasty Register. Clin Epidemiol 2016; 8: 509-14. doi: 102147/ clepS99498 
12. Statistics Denmark. About us. Available from: https://www.dst.dk/en/omDS

13. Pottegård A, Schmidt S A J, Wallach-Kildemoes H, Sørensen H T, Hallas J, Schmidt M. Data resource profile: the Danish National Prescription Registry. Int J Epidemiol 2017; 46: 798-798f. doi: 101093/ije/ dyw 213

14. Schmidt M, Pedersen L, Sorensen H T. The Danish Civil Registration System as a tool in epidemiology. Eur J Epidemiol 2014; 29: 541-9. doi: 101007/s10654-014-9930-3

15. Schmidt M, Schmidt S A, Sandegaard J L, Ehrenstein V, Pedersen L, Sorensen H T. The Danish National Patient Registry: a review of content, data quality, and research potential. Clin Epidemiol 2015; 7: 449-90. doi: $102147 /$ clepS91125

16. Schmolders J, Friedrich M J, Michel R, Strauss A C, Wimmer M D, Randau T M, et al. Validation of the Charlson comorbidity index in patients undergoing revision total hip arthroplasty. Int Orthop 2015; 39: 1771-7. doi: 101007/s00264-015-2810-y

17. StataCorp. Stata: Release 16 Statistical Software. College Station, TX: StataCorp LLC; 2019.

18. Cook D J, Kaskovich S W, Pirkle S C, Mica M A C, Shi L L, Lee M J. Benchmarks of duration and magnitude of opioid consumption after total hip and knee arthroplasty: a database analysis of 69,368 patients. J Arthroplasty 2019; 34: 638-644e631. doi: 101016/jarth201812023

19. Danish Health Authority. Opioidbehandling af kroniske non-maligne smerter 2018. Sundhedsstyrelsen-prod-184570-cd.azurewebsites.net

20. Hernández C, Díaz-Heredia J, Berraquero M L, Crespo P, Loza E, Ruiz Ibán M. Pre-operative predictive factors of post-operative pain in patients with hip or knee arthroplasty: a systematic review. Reumatol Clin 2015; 11: 361-80. doi: 101016/jreuma201412008

21. Grol-Prokopczyk H. Use and opinions of prescription opioids among older American adults: sociodemographic predictors. J Gerontol B Psychol Sci Soc Sci 2019; 74: 1009-19. doi: 101093/geronb/gby093

22. Klüwer-Trotter B, Lian O S. Attitudes to seeking medical assistance: variations depending on social background? Tidsskr Nor Laegeforen 2012; 132: 36-40. doi: 104045/tidsskr110380

23. Vyas M V, Watt J A, Yu A Y X, Straus S E, Kapral M K. The association between loneliness and medication use in older adults. Age Ageing 2021; 50: 587-91. doi: 101093/ageing/afaa177

24. Roberts K C, Moser S E, Collins A C, McCardel B R, Schultz K A, Schaffer N E, et al. Prescribing and consumption of opioids after primary, unilateral total hip and knee arthroplasty in opioidnaive patients. J Arthroplasty 2020; 35: 960-965e961. doi: 101016/ jarth201908036

25. Schneeweiss S, Avorn J. A review of uses of health care utilization databases for epidemiologic research on therapeutics. J Clin Epidemiol 2005; 58: 323-37. doi: 101016/jjclinepi200410012

26. Hunter D J, Bierma-Zeinstra S. Osteoarthritis. Lancet 2019; 393: 1745-59. doi: 101016/s0140-6736(19)30417-9

27. Keurentjes J C, Fiocco M, So-Osman C, Onstenk R, Koopman-Van Gemert A W, Pöll R G, et al. Patients with severe radiographic osteoarthritis have a better prognosis in physical functioning after hip and knee replacement: a cohort-study. PLoS One 2013; 8: e59500. doi: 101371/ journalpone0059500 


\section{Supplementary data}

Table 1. Names and ATC codes for included medications

\begin{tabular}{|c|c|c|c|}
\hline \multicolumn{2}{|l|}{ NSAID } & \multicolumn{2}{|l|}{ Opioids } \\
\hline Phenylbutazone & M01AA01 & Morphine & N02AA01 \\
\hline Indomethacin & M01AB01 & Hydromorphone & N02AA03 \\
\hline Sulindac & M01AB02 & Nicomorphine & N02AA04 \\
\hline Tolmetin & M01AB03 & Oxycodone & N02AA55 \\
\hline Diclofenac & M01AB05 & Pethidine & N02AB02 \\
\hline Etodolac & M01AB08 & Fentanyl & N02AB03 \\
\hline Ketorolac & M01AB15 & Buprenorphine & N02AE01 \\
\hline Aceclofenac & M01AB16 & Ketobemidone & N02AG02 \\
\hline Diclofenac & M01AB55 & Codeine and paracetamol & N02AJ06 \\
\hline Piroxicam & M01AC01 & Tramadol & N02AX02 \\
\hline Tenoxicam & M01AC02 & Tapentadol & N02AX06 \\
\hline Lornoxicam & M01AC05 & Methadone & N07BC02 \\
\hline Meloxicam & M01AC06 & Codeine & R05DA04 \\
\hline Ibuprofen & M01AE01 & & \\
\hline Naproxen & M01AE02 & & \\
\hline Ketoprofen & M01AE03 & & \\
\hline Fenoprofen & M01AE04 & & \\
\hline Fenbufen & M01AE05 & & \\
\hline Flurbiprofen & M01AE09 & & \\
\hline Surgamyl & M01AE11 & & \\
\hline Dexibuprofen & M01AE14 & & \\
\hline Dexketprofen & M01AE17 & & \\
\hline Naproxen (+esomeprazol) & M01AE52 & & \\
\hline Celecoxib & M01AH01 & & \\
\hline Rofecoxib & M01AH02 & & \\
\hline Etoricoxib & M01AH05 & & \\
\hline Nabumeton & M01AX01 & & \\
\hline Glucosamine & M01AX05 & & \\
\hline
\end{tabular}

Due to the time span of the study (1996-2018) some medication types are included despite being out-dated in 2021 